\title{
Fluid flow between active elastic plates
}

\author{
D. V. Strunin ${ }^{1}$
}

(Received 15 August 2008; revised 01 April 2009)

\begin{abstract}
We propose a model of a channel flow between actively moving elastic plates as a framework for blood flow in active human arteries. The main difference from extant models is that our model is autonomous. It is a nonlinear partial differential equation governing the deformation of the plates involving sixth order spatial derivative. The equation has a similar structure to the model we proposed earlier to simulate another active system-spinning combustion front.
\end{abstract}

\section{Contents}

1 Introduction

C872

2 Pulses as auto-waves

4 Passive elastic walls

http://anziamj.austms.org.au/ojs/index.php/ANZIAMJ/article/view/1452 gives this article, (C) Austral. Mathematical Soc. 2009. Published May 22, 2009. ISSN 1446-8735. (Print two pages per sheet of paper.) 


\section{Introduction}

The overwhelming majority of mathematical models of arterial blood flow treat arteries as passive material [1,2]. A popular approximation of the flow-artery interaction is a proportional increase of the artery's cross-section to an increase of the flow pressure [3]

$$
p-p_{0} \sim \sqrt{\AA}-\sqrt{A_{0}},
$$

where $p_{0}$ and $A_{0}$ are the reference pressure and cross-sectional area respectively. The works focused on pulse modelling [4] interpret pulses as a passive response to time-periodic boundary condition imposed at the artery's inlet.

However, the arteries have muscles which actively push the blood. In some models [5] the arteries do actively exert pressure. However, such models are non-autonomous, that is the active component of the pressure, despite in place, is introduced by an explicit function of time and coordinate. Consequently, the pulses take place because this function already has the form of pulses. Effectively it represents some 'outside controller' dictating the system's behaviour.

This note proposes an approach which leads to an autonomous model of the flow between hypothetically active elastic walls. At this stage we do not claim direct connection with biology, but aim to demonstrate the possibility of constructing such a model. 


\section{Pulses as auto-waves}

Two crucial factors governing the blood flow are pressure gradient and viscous resistance from the walls. Due to the viscosity our model will be dissipative. Consequently, the pulses will be auto-waves; that is, the pulses are self-sustained dissipative structures. In distinction from conservative waves, such as those on water, the auto-waves propagate with unique speed and amplitude.

Previously we formulated the following auto-wave model for spinning combustion fronts [6]:

$$
\frac{\partial F}{\partial t}=\frac{\partial^{6} F}{\partial x^{6}}-\frac{\partial}{\partial x}\left[\left(\frac{\partial F}{\partial x}\right)^{3}\right]+\left(\frac{\partial F}{\partial x}\right)^{4} .
$$

Here $F(x, t)$ stands for the position of the front subject to periodic boundary conditions. The front is thought to be a surface (line in 1D case) separating hot burned products from cold fresh composition. It is important to note that the combustion system is active and dissipative: its activity is due to heat generation in a chemical reaction; and dissipation is due to heat conductivity. These features place the combustion fronts and the arterial blood flow into the same category of active dissipative systems.

By differentiating with respect to $x$ we rewrite equation (1) in terms of the derivative $\partial F / \partial x \equiv W$ :

$$
\frac{\partial W}{\partial t}=\frac{\partial^{6} W}{\partial x^{6}}-\frac{\partial^{2}}{\partial x^{2}}\left(W^{3}\right)+\frac{\partial}{\partial x}\left(W^{4}\right) .
$$

A spatially uniform solution $F=$ const of (1) and corresponding uniform solution $W \equiv 0$ of $(2)$ are stable under small perturbations as the linearised equation, $\partial F / \partial t=\partial^{6} F / \partial x^{6}$, is purely dissipative.

For brevity, from now on we use primes and Roman numerals to denote derivatives on $x$. 

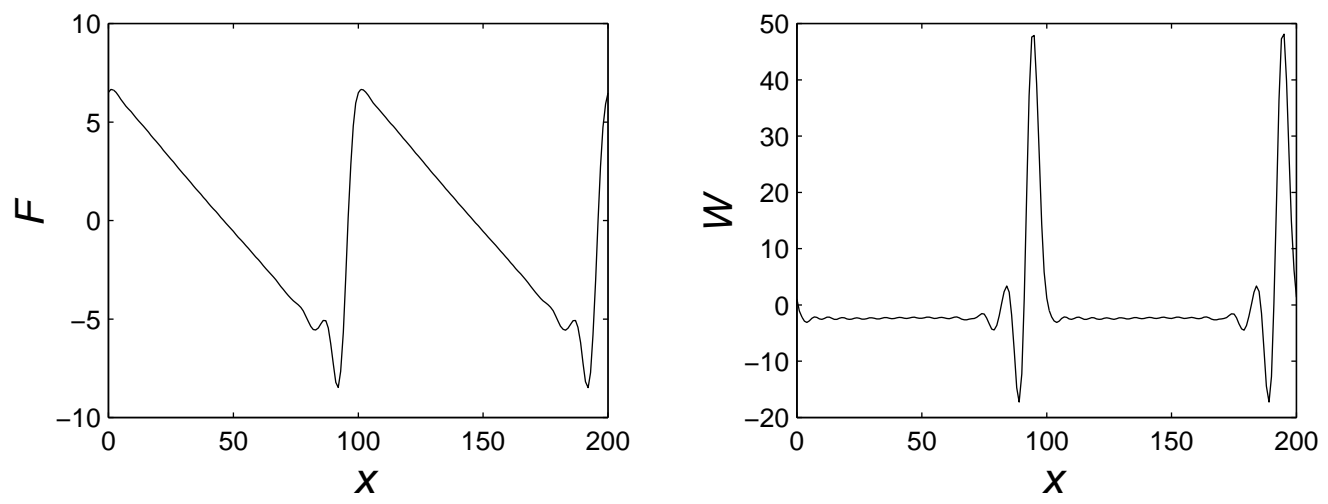

Figure 1: A train of kink shaped (F) and pulse shaped (W) auto-waves. The F-wave moves upwards and to the left; the $\mathrm{W}$-wave moves horizontally to the left.

To explain the mechanism of (1), denote a typical amplitude of the perturbation of the uniform solution by $\Delta \mathrm{F}>0$ and a typical spatial scale of the perturbation by $\Delta x>0$. If the perturbation is sufficiently large, it grows because of the pumping effect of the (third order) nonlinear source, $-\left(\mathrm{F}^{\prime 3}\right)^{\prime}=\left(-3 \mathrm{~F}^{\prime 2}\right) \mathrm{F}^{\prime \prime}$. Effectively, this is an anti-diffusion term with the negative multiplier in front of $F^{\prime \prime}$. Evaluate the terms by the order of magnitude in absolute value. We have

$$
\left(\mathrm{F}^{\prime 3}\right)^{\prime} \sim(\Delta \mathrm{F})^{3} /(\Delta \mathrm{x})^{4} .
$$

As the perturbation grows, the higher order nonlinearity comes into play,

$$
\left(\mathrm{F}^{\prime}\right)^{4} \sim(\Delta \mathrm{F})^{4} /(\Delta \mathrm{x})^{4} .
$$

It acts so that certain sections of the $\mathrm{F}$ profile become steeper (the $\mathrm{W}$ profile locally surges in amplitude) making $\Delta x$ small. On those sections the dissipation prevails because it is of higher order in $\Delta x$,

$$
\mathrm{F}^{\mathrm{VI}} \sim \Delta \mathrm{F} /(\Delta \mathrm{x})^{6} .
$$


As a result, the steep sections are smoothed out and the perturbation, instead of turning into a singularity, becomes a smooth self-sustained dissipation structure. It has the form of a train of pulses shown in Figure 1. Each individual pulse is essentially a stable auto-soliton with the amplitude and speed controlled by the dynamic equation, not the initial condition.

We refer to an analogy with damping in a viscous flow between elastic walls. It is known that dissipation for such a flow is represented by a sixth order spatial derivative $[7,8]$. This brings an idea to extend the model [7] by source terms representing active motion of the elastic walls in order to make the model structurally similar to (2).

\section{Lubrication model for the flow}

Consider the flow between elastic walls, assuming symmetry with respect to the middle plane, $z=0$; hence it suffices to analyse only half of the flow, $0<z<\mathrm{H}(\mathrm{t})$.

Adopting lubrication theory [7] we equate the pressure gradient to viscous friction:

$$
\frac{\partial^{2} v}{\partial z^{2}}=\frac{1}{\eta} \frac{\partial p}{\partial x},
$$

where $x$ and $z$ are the coordinates along and across the flow respectively, $v(x, z, t)$ is the flow velocity in the $x$ direction, $p(x, t)$ is the pressure, and $\eta$ is the viscosity.

The pressure is assumed $z$-independent, so that integrating (3) on $z$ gives

$$
v=\frac{1}{2 \eta} \frac{\partial p}{\partial x}\left(z^{2}-H^{2}\right)+v(x, H, t) .
$$

The mass flux is

$$
\mathrm{Q}=\int_{0}^{\mathrm{H}} v \mathrm{~d} z=-\frac{\mathrm{H}^{3}}{3 \eta} \frac{\partial p}{\partial x}+v(x, H, t) H .
$$


Define the transversal displacement of the wall, $w(x, t)$, from the neutral position, $\mathrm{H}_{0}$, by

$$
\mathrm{H}=\mathrm{H}_{0}+w .
$$

Then the continuity equation becomes

$$
\frac{\partial w}{\partial t}+\frac{\partial Q}{\partial x}=0
$$

Substituting (5) into (7) gives

$$
\frac{\partial w}{\partial t}=\frac{\partial}{\partial x}\left[\frac{H^{3}}{3 \eta} \frac{\partial p}{\partial x}-v(x, H, t) H\right] .
$$

\section{Passive elastic walls}

Equation (8) links the displacement of the flow boundary, coinciding with the wall, to the flow pressure. Elasticity theory $[9,10]$ provides the reverse link from the pressure to the displacement

$$
p=D \frac{\partial^{4} w}{\partial x^{4}}-\frac{\partial}{\partial x}\left(N \frac{\partial w}{\partial x}\right)
$$

where

$$
N=\frac{E h}{1-v^{2}}\left[\frac{\partial u}{\partial x}+\frac{1}{2}\left(\frac{\partial w}{\partial x}\right)^{2}\right]
$$

In (9) and (10) $\mathfrak{u}(\boldsymbol{x}, \mathbf{t})$ is the wall's displacement along the flow, D is the flexural rigidity of the wall, $E$ is Young's modulus, $h$ is the thickness of the wall, $v$ is Poisson's ratio, and $\mathrm{N}$ is the force caused by the displacements.

Substituting (10) and (9) into (8), and using the no-slip boundary condition,

$$
v(x, H, t)=\frac{\partial u}{\partial t},
$$


we obtain

$$
\begin{aligned}
\frac{\partial w}{\partial t}= & \frac{D}{3 \eta}\left(H^{3} w^{v}\right)^{\prime}-\frac{E h}{6 \eta\left(1-v^{2}\right)}\left[H^{3}\left(w^{\prime 3}\right)^{\prime \prime}\right]^{\prime} \\
& -\frac{E h}{3 \eta\left(1-v^{2}\right)}\left[H^{3}\left(u^{\prime} w^{\prime}\right)^{\prime \prime}\right]^{\prime}-\left(\frac{\partial u}{\partial t} H\right)^{\prime} .
\end{aligned}
$$

The shear stress in the fluid is $T=\eta \partial v / \partial z$, therefore on the boundary, $z=\mathrm{H}$, using (4),

$$
\mathrm{T}=\mathrm{p}^{\prime} \mathrm{H} .
$$

This shear stress must be equal to the shear stress produced by the wall,

$$
\mathrm{T}=\mathrm{N}^{\prime} \text {. }
$$

Equating (12) and (13) with the use of (10), we have

$$
\frac{E}{1-v^{2}}\left[u^{\prime \prime}+\frac{1}{2}\left(w^{\prime 2}\right)^{\prime}\right]=p^{\prime} H .
$$

The three equations (11), (14) and (9) form the closed system with respect to the three unknown functions $w(x, t), \mathfrak{u}(x, t)$ and $p(x, t)$.

Now solve the system for small amplitude perturbations. They satisfy the linearised equations

$$
\begin{aligned}
& \frac{E}{1-v^{2}} u^{\prime \prime}=D H_{0} w^{v}, \\
& \frac{\partial w}{\partial t}=\frac{H_{0}^{3}}{3 \eta} D w^{V I}-\frac{\partial u^{\prime}}{\partial t} H_{0} .
\end{aligned}
$$

Looking for solutions

$$
w=A(t) \sin (k x), \quad u=B(t) \cos (k x),
$$

we obtain

$$
\frac{E}{1-v^{2}} B=-D_{0} k^{3} A
$$




$$
\frac{d A}{d t}=-\frac{H_{o}^{3}}{3 \eta} D k^{6} A+H_{0} k \frac{d B}{d t} .
$$

It is easy to deduce that the amplitudes decay:

$$
A(t) \sim B(t) \sim \exp \left[-\frac{H_{0}^{3} D E k^{6}}{3 \eta E+3 \eta D H_{0}^{2}\left(1-v^{2}\right) k^{4}} t\right] .
$$

\section{$5 \quad$ Active elastic walls}

We intend to construct a model assuming that the walls actively move. Suppose that, when deflected from the neutral position, they exert the extra pressure relative to (9),

$$
\mathrm{p}=\mathrm{D} w^{\mathrm{IV}}-\left(\mathrm{N} w^{\prime}\right)^{\prime}+\mathrm{p}_{1}
$$

where $p_{1}$ depends on $w$. We postulate that $p_{1}$ is proportional to the fourth power of the vertical displacement (this leads to an analogy with the term $W^{3}$ in (2)),

$$
p_{1}=-\alpha w^{4}, \quad \alpha>0 .
$$

Further, suppose that the walls actively move along the flow, thereby producing extra shear stress relative to (14). We postulate that the wall's motion along the flow, represented by the displacement $u$ and velocity $\partial u / \partial t$, is coupled with $w$. Specifically, the $\mathrm{H}$-weighted velocity along the flow, $\mathrm{H} \partial \mathrm{u} / \partial t$, combined with the other $\mathfrak{u}$-containing term in (11), is proportional to the fifth power of $w$ (this leads to an analogy with the term $W^{4}$ in (2)),

$$
-\frac{E h}{3 \eta\left(1-v^{2}\right)} H^{3}\left(u^{\prime} w^{\prime}\right)^{\prime \prime}-\frac{\partial u}{\partial t} H=\beta w^{5}, \quad \beta>0 .
$$

From a physical viewpoint this relation should reflect an extra (active) shear stress, which we denote $T_{1}$. The total stress satisfies the continuity condition on the boundary

$$
\mathrm{T}_{1}+\frac{\mathrm{E}}{1-v^{2}}\left[\mathrm{u}^{\prime \prime}+\frac{1}{2}\left(w^{\prime 2}\right)^{\prime}\right]=\mathrm{p}^{\prime} \mathrm{H}
$$


where the pressure $p$ is represented by (16).

Although we are not able to justify specific powers used in (17) and (18), these laws express a biologically motivated property of larger active response from the walls to larger deformations $w$.

Under the assumptions (16), (17) and (18), the equation (11) governing the dynamics of the vertical displacement becomes $\boldsymbol{u}$-independent:

$$
\begin{aligned}
\frac{\partial w}{\partial t}= & \frac{D}{3 \eta}\left(H^{3} w^{v}\right)^{\prime}-\frac{E h}{6 \eta\left(1-v^{2}\right)}\left[H^{3}\left(w^{\prime 3}\right)^{\prime \prime}\right]^{\prime} \\
& -\frac{1}{3 \eta} \alpha\left[H^{3}\left(w^{4}\right)^{\prime}\right]^{\prime}+\beta\left(w^{5}\right)^{\prime} .
\end{aligned}
$$

We arrived at the closed system. The procedure of finding solutions is as follows. The function $w(x, t)$ is obtained from (20) under, say, periodic boundary conditions. Then $\mathfrak{u}(x, t)$ is found from (18) and the pressure from (16) and (17). The extra shear stress $T_{1}(x, t)$ and total stress are obtained from (19).

Let us analyse the mechanism of (20) in the same way as we did for the combustion equations. Transform (20) into the equation with respect to $f$ defined by $w=f^{\prime}$,

$$
\frac{\partial f}{\partial t}=\frac{D}{3 \eta} H^{3} f^{V I}-\frac{E h}{6 \eta\left(1-v^{2}\right)} H^{3}\left[\left(f^{\prime \prime}\right)^{3}\right]^{\prime \prime}-\frac{1}{3 \eta} \alpha H^{3}\left[\left(f^{\prime}\right)^{4}\right]^{\prime}+\beta\left(f^{\prime}\right)^{5} .
$$

Note that the first two terms in the right-hand side come with the classical theory and, therefore, are dissipative.

Comparing with (1), note the higher order of nonlinearity of the source (fourth instead of third); this is necessary to overpower the third order classical term in (21). Accordingly, the last term in (21) has even higher, fifth, order of nonlinearity, required to counterbalance the source as the perturbation grows. 
Evaluate the terms by the order of magnitude in absolute value. The source,

$$
\frac{1}{3 \eta} \alpha H^{3}\left[\left(f^{\prime}\right)^{4}\right]^{\prime} \sim\left(f^{\prime}\right)^{3} f^{\prime \prime} \sim(\Delta f)^{4} /(\Delta x)^{5} .
$$

As the amplitude of the perturbation increases, the fifth order nonlinearity comes into play,

$$
\beta\left(f^{\prime}\right)^{5} \sim(\Delta f)^{5} /(\Delta x)^{5} .
$$

It steepens the profile of $f$ at certain sections where the dissipation eventually prevails due to higher order in $\Delta x$,

$$
\frac{\mathrm{D}}{3 \eta} \mathrm{H}^{3} \mathrm{f}^{\mathrm{VI}} \sim \Delta \mathrm{f} /(\Delta \mathrm{x})^{6} .
$$

This mechanism is similar to that of (1), so pulse waves should exist in the hydro-elastic model.

\section{Discussion}

Our model meets the two major requirements: autonomy and capacity to produce pulses as auto-waves. The pulses are a corollary of the assumption that the walls actively push the fluid against viscous forces.

A living organism is a self-organised system. The property of our model to exhibit, in contrast to all other biofluid models, a self-organised behaviour is new in pulse modelling. It remains to be seen whether or not this approach leads to any practical modelling. However, we predict, based on the classical mechanical laws employed, that, if the walls are coupled with the flow exactly as prescribed by (16) and (18), then the pulses would occur. It is probably possible to set up a verifying experiment, although it may not be technically simple. 
An important issue is the self-consistency of the model. It should guarantee that overall displacement of the arteries over some period of time, $\tau$, is zero,

$$
\int_{\tau} \frac{\partial u}{\partial t} d t=0, \quad \int_{\tau} \frac{\partial w}{\partial t} d t=0 .
$$

In the proposed model the zero value of the transversal displacement, $w$, is guaranteed by periodicity of $w$. Indeed, the wave solution depends on $x / c+t$, where $c$ is the wave speed. Integrating equation (20) on $t$ over the wave period, or many periods, is equivalent to integrating on $x / c$. This gives zero because all the terms in the equation's right-hand side are full derivatives and the expressions under them are periodic.

Periodicity of the displacement along the flow, $\mathfrak{u}$, is more difficult to achieve. In view of (18), the first condition (22) becomes

$$
\int_{\tau}\left[\frac{E h}{3 \eta\left(1-v^{2}\right)} H^{2}\left(u^{\prime} w^{\prime}\right)^{\prime \prime}+\frac{\beta}{H} w^{5}\right] d t=0 .
$$

At this stage of the modelling we use the value of parameter $\beta$ that is at our disposal. In order to satisfy condition (23) one needs to tune $\beta$.

Note that, unlike the combustion model (2), the hydro-elastic model (20) generates only pulses travelling to the left. This is caused by asymmetry: the source term acts as a source only on sections with positive slope, $f^{\prime}>$ 0 , because on those sections, $-\left(f^{\prime}\right)^{3} f^{\prime \prime}$ is effectively an anti-diffusion with negative coefficient, $-\left(f^{\prime}\right)^{3}<0$, in front of $f^{\prime \prime}$, whereas on negative slopes, $f^{\prime}<0$, it acts as diffusion. Yet, we are satisfied with this property as it implies that our quasi-artery 'knows' the direction to the 'heart', which would be to the right in Figure 1, and therefore generates pulses travelling in the opposite direction, to the left.

Our next remark goes to the expressions adopted for the extra pressure (17) and extra shear stress (18)-(19). They are empirical and contain the unknown coefficients $\alpha$ and $\beta$. The fact that we have only two of them is 
rather positive because for complex systems, such as biological, the more details one takes into account the more coefficients need to be involved. Values of such coefficients are difficult to determine.

\section{Conclusion}

We presented an autonomous model, without directly linking to biology at this stage, of a hypothetical flow between active walls. Lubrication theory is used for the flow, and the walls are supposed to actively exert pressure and shear stress. The analogy with the combustion front equations indicates that the model should have auto-wave solutions in the form of pulses.

\section{References}

[1] Sherwin, S. J., Formaggia, L., Peiro, J. and Franke, V., Computational modelling of $1 \mathrm{D}$ blood flow with variable mechanical properties and its application to the simulation of wave propagation in the human arterial system, Int. J. Numer. Meth. Fluids, 43, 2003, 673-700. doi:10.1002/fld.543 C872

[2] C. Kleinstreuer. Biofluid Dynamics. Taylor and Francis. Boca Raton, 2006. C872

[3] Olufsen, M. S., Peskin, C. S., Kim W. Y., Pedersen, E. M., Nadim, A. and Larsen, J., Numerical simulation and experimental validation of blood flow in arteries with structured-tree outflow conditions, Annals of Biomedical Engineering, 28, 2000, 1281-1299. doi:10.1114/1.1326031 C872

[4] Matthys, K. S., Alastruey, J., Peiro, J., Khir, A. W., Segers, P., Verdonck, P. R., Parker, K. H. and Sherwin, S. J., Pulse wave 
propagation in a model human arterial network: Assessment of 1-D numerical simulations against in vitro measurements, J. Biomech., 40, 2007, 3476-3486. doi:10.1016/j.biomech.2007.05.027 C872

[5] A. J. Roberts. A One-dimensional Introduction to Continuum Mechanics. World Scientific. Singapore, 1994. C872

[6] Strunin, D. V., Autosoliton model of the spinning fronts of reaction, IMA J. Appl. Math., 63, 1999, 163-177. doi:10.1093/imamat/63.2.163 C873

[7] Huang, R. and Suo, Z., Wrinkling of a compressed elastic film on a viscous layer, J. Appl. Phys., 91, 2002, 1135-1142. doi:10.1063/1.1427407 C875

[8] King, J. R., The isolation oxidation of silicon: the reaction-controlled case, SIAM J. Appl. Math., 49, 1989, 1064-1080. doi:10.1137/0149064 C875

[9] L. D. Landau and E. M. Lifshitz. Theory of Elasticity. Pergamon. London, 1959, 57-60. C876

[10] S. Timoshenko and S. Woinowsky-Krieger. Theory of Plates and Shells. McGraw-Hill. New York, 1987. C876

\section{Author address}

1. D. V. Strunin, Dept. Maths \& Computing, University of Southern Queensland, Toowoomba, Australia. mailto: strunin@usq. edu. au 